\title{
Chain dynamics in the low-temperature phases of lipid membranes by electron spin-echo spectroscopy
}

\author{
Rosa Bartucci, ${ }^{\mathrm{a}, *}$ Rita Guzzi, ${ }^{\mathrm{a}}$ Derek Marsh, ${ }^{\mathrm{b}}$ and Luigi Sportelli ${ }^{\mathrm{a}}$ \\ ${ }^{a}$ Dipartimento di Fisica and Unità INFM, Università della Calabria, I-87036 Arcavacata di Rende (CS), Italy \\ b Max-Planck-Institut für Biophysikalische Chemie, Abteilung Spektroskopie, 37077 Göttingen, Germany
}

Received 21 October 2002; revised 20 December 2002

\begin{abstract}
Spin-echo decays of spin-labelled phospholipids have been recorded to study the chain dynamics in the low-temperature phases of dipalmitoyl phosphatidylcholine membranes with and without $50 \mathrm{~mol} \%$ cholesterol. The phase-memory relaxation time, $T_{2 \mathrm{M}}$, depends on the position of spin-labelling in the $s n-2$ chain, and on the presence of cholesterol. A biphasic temperature dependence of $T_{2 \mathrm{M}}$ is obtained over the range 150-270 K. Echo-detected field-swept absorption EPR spectra were recorded as a function of the echo delay time, $\tau$. The echo-detected EPR lineshapes show a strong dependence on $\tau$, revealing anisotropic phase relaxation arising from torsional chain motions. Cholesterol has a large effect on torsional oscillations about the chain long axis. Small-amplitude chain motions in the low-temperature phases may be important for cryopreservation of membranes.
\end{abstract}

(C) 2003 Elsevier Science (USA). All rights reserved.

Keywords: Lipid bilayer; Spin label; EPR; Electron spin echo; Chain librations; Cryoprotection

\section{Introduction}

Spin-label electron paramagnetic resonance (EPR) spectroscopy has proved to be a most valuable means for investigating rotational dynamics in biological systems (see e.g., $[1,2]$ ). A particularly rewarding application of the technique is to the study of biological membranes (see e.g., [3,4]). Routinely, continuous wave (CW) EPR spectroscopy - both conventional and saturation-transfer, depending on the timescale - is used to obtain lineshapes of nitroxide spin labels that are sensitive to rotational motion $[5,6]$.

In principle, time-resolved pulsed EPR techniques offer a more direct approach to studying spin-label motions. Electron spin-echo measurements of the phasememory time, $T_{2 \mathrm{M}}$, are related directly to rotational correlation times in dynamic systems [7]. Furthermore, the partially relaxed, echo-detected EPR lineshapes display a dependence on echo delay time, $\tau$, that is characteristic of small-amplitude angular motions $[8,9]$. Dzuba and colleagues $[10,11]$ have characterised the

\footnotetext{
${ }^{*}$ Corresponding author. Fax: +39-0984-494401.

E-mail address: bartucci@fis.unical.it (R. Bartucci).
}

echo-detected spectra of small spin-label probes in glassy solvents as being attributable to librational motions. This provides a framework in which to interpret similar spectra from spin-labelled lipids in the lowtemperature phases of biological membranes. Some measurements of the phase-memory time, $T_{2 \mathrm{M}}$, have already been reported for spin-labelled cholestane and spin-labelled phosphatidylcholine in oriented membranes at low hydration [12].

In this paper, we present a systematic study of spinlabelled phosphatidylcholines in fully hydrated lipid membranes. We investigate the effects of label position in the $s n-2$ chain, of varying temperature and of cholesterol on the dynamics of lipid chain motion in the low-temperature regime. Measurements are made both of $T_{2 \mathrm{M}}$ from echo decays and of field-swept EPR spectra that are detected from the echo maxima recorded at different fixed values of the echo delay time, $\tau$.

\section{Experimental}

Dipalmitoyl phosphatidylcholine (DPPC) and cholesterol were from Sigma/Aldrich (St. Louis, MO). 
Spin-labelled phosphatidylcholines (1-acyl-2-(n-doxylstearoyl)-sn-glycero-3-phosphocholine, $n$-PCSL) were from Avanti Polar lipids (Birmingham, AL) or synthesised according to Marsh and Watts [13].

DPPC with $1 \mathrm{~mol} \%$ of $n$-PCSL, with and without $50 \mathrm{~mol} \%$ cholesterol, were codissolved in chloroform. Solvent was evaporated with a nitrogen gas stream and residual traces removed by drying under vacuum overnight. The lipid $(15 \mathrm{mg})$ was dispersed in phosphatebuffered saline (PBS) at $\mathrm{pH} 7.5$ at a concentration of ca. $100 \mathrm{mg} / \mathrm{ml}$ by vortex mixing with heating to $60^{\circ} \mathrm{C}$, i.e., above the chain-melting phase transition. The sample was then transferred to a standard $4 \mathrm{~mm}$-diameter, quartz EPR tube, concentrated by pelletting in a benchtop centrifuge and the excess supernatant removed.

Data were collected on a Elexsys E580 $9 \mathrm{GHz}$ FT-EPR spectrometer (Bruker, Karlsruhe, Germany) equipped with a Flexline MD5, variable-Q, dielectric resonator and a nitrogen gas-flow temperature control unit. Two-pulse, Hahn-echo decays were obtained by using microwave pulse widths of 12 and $24 \mathrm{~ns}$, with the microwave power adjusted to give $\pi / 2$ and $\pi$-pulses, or less, respectively. The interpulse spacing was incremented from $\tau=88 \mathrm{~ns}$ in $4 \mathrm{~ns}$ steps. The phase-memory time, $T_{2 \mathrm{M}}$, was determined by fitting the maximum echo amplitude as a function of $2 \tau$ with a simple exponential decay. Echo-detected absorption EPR spectra were obtained by recording the echo maximum, whilst sweeping the magnetic field. The interpulse spacing $\tau$ was chosen to coincide with the progressive minima of the proton modulation in the echo decay.

Integration of the echo, rather than recording the maximum, could avoid potential broadening by offresonance oscillations of the echo. However, the spectra are powder lineshapes from randomly oriented membrane dispersions, and therefore intrinsically contain a large degree of orientational spectral overlap.

\section{Results}

\subsection{Echo decay curves and $T_{2 M}$}

Fig. 1 gives typical echo decay curves as a function of interpulse spacing, $\tau$, for 14-PCSL in membranes of dipalmitoyl phosphatidylcholine at different temperatures. The spectrometer field is fixed at the position corresponding to the maximum amplitude of the central line in the EPR spectrum for all measurements. High frequency modulation of the echo decay arises from the interaction of the unpaired electron spin of the nitroxide-labelled lipid with the nuclear spin of nearby bonded protons. The effect of lipid chain dynamics on the decay curves is very evident from the pronounced temperature dependence. An approximately single exponential decay of the maximum echo amplitude is obtained in each

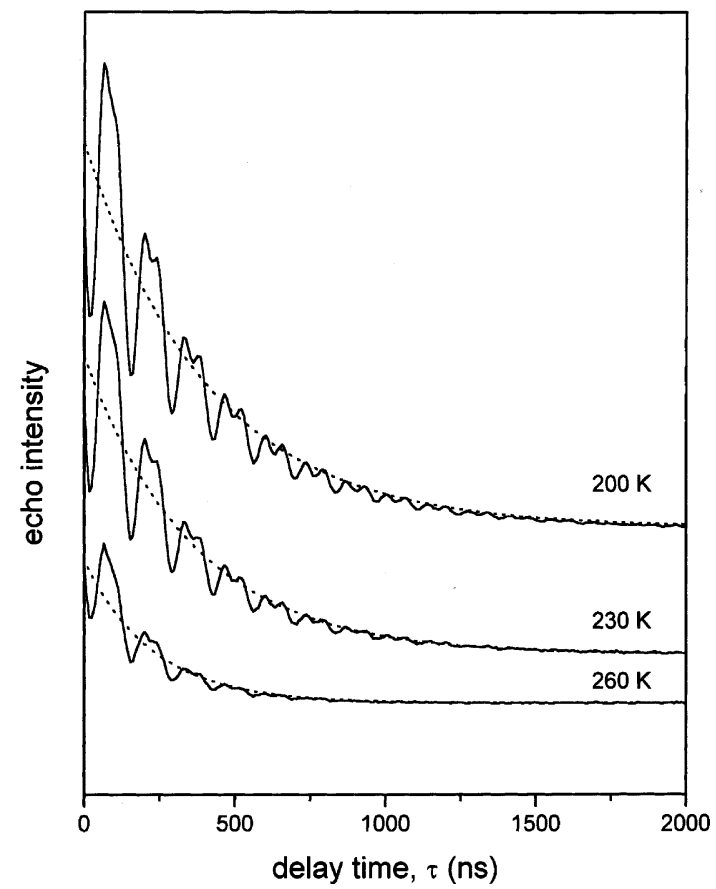

Fig. 1. Representative echo decay curves, as a function of delay time, $\tau$, for the 14-PCSL spin label in dipalmitoyl phosphatidylcholine bilayers. Sample temperatures are indicated on the figure. The dashed lines are fits with a single exponential decay function.

case. The decay rate characterised by the phase-memory time, $T_{2 \mathrm{M}}$, increases with increasing temperature. Addition of $50 \mathrm{~mol} \%$ cholesterol to the membranes causes an increase in decay rate in most cases (data not shown).

Fig. 2 gives the temperature dependence of the phasememory time, $T_{2 \mathrm{M}}$, which was determined by fitting a single exponential to each echo decay (see Fig. 1). The maximum temperature for which echoes of reasonable intensity could be recorded depends on sample and spinlabel position. It was never greater than $280 \mathrm{~K}$. Data are given for various $n$-PCSL spin labels in membranes of DPPC (Fig. 2a) and DPPC $+50 \mathrm{~mol} \%$ cholesterol (Fig. 2b). In general, a biphasic temperature dependence is obtained, although for 5-PCSL (and possibly also for 7PCSL) the temperature at which $T_{2 \mathrm{M}}$ reaches its maximum value lies at or below the lowest temperature of measurement (viz., $150 \mathrm{~K}$ ). The data for the different $n$-PCSL positional isomers tend to fall into groups, at least for membranes of DPPC $+50 \mathrm{~mol} \%$ cholesterol. $T_{2 \mathrm{M}}$ for 5-PCSL and 7-PCSL has rather similar values and temperature dependence; 10-PCSL and 12-PCSL also group together, and 14-PCSL reaches the highest $T_{2 \mathrm{M}}$ values (Fig. 2b). Somewhat similar trends are found for membranes of DPPC alone, except that 10-PCSL has anomalously low values of $T_{2 \mathrm{M}}$ (Fig. 2a). The latter exception can be explained by partial segregation of the spin label in the low-temperature phase. This results in spin-spin interactions that are evident as a strong broadening of the conventional EPR spectrum (not 

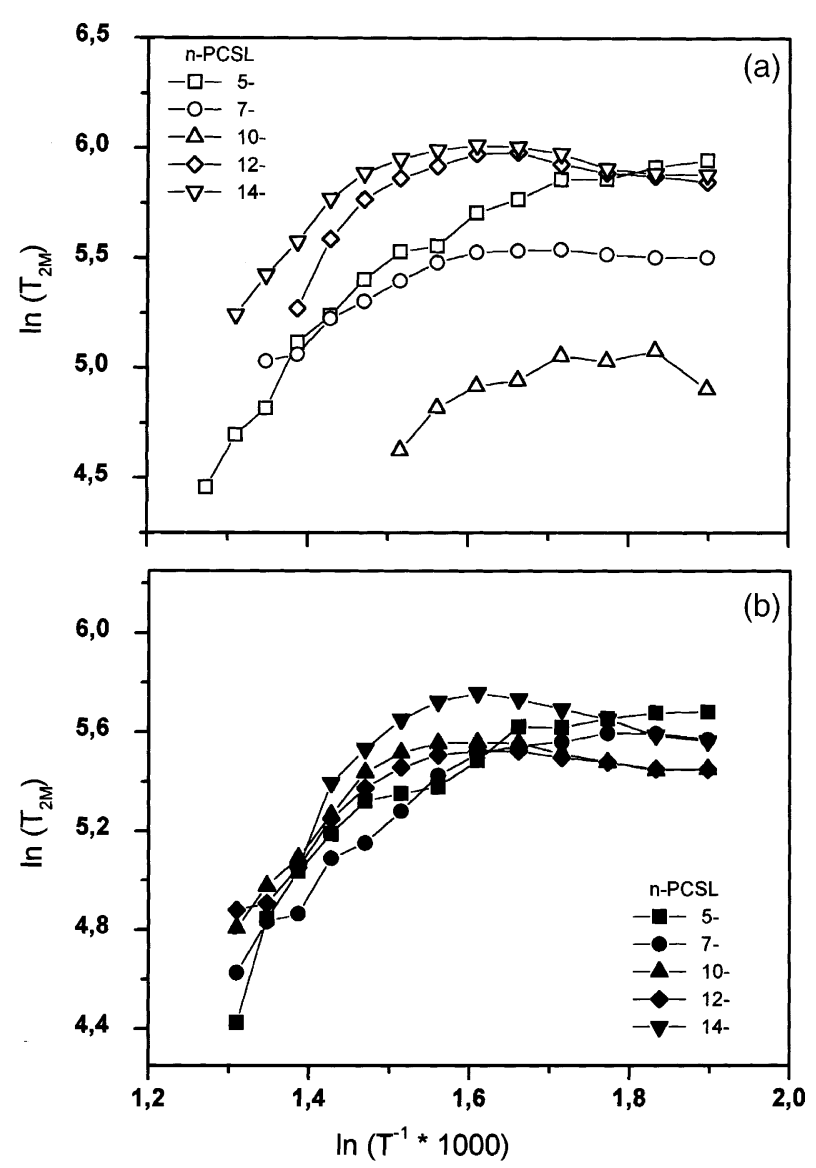

Fig. 2. Dependence on temperature, $T$, of the phase-memory time, $T_{2 \mathrm{M}}$, of the electron spin-echo decays from spin-labeled phosphatidylcholine, $n$-PCSL, in membranes of: (a) dipalmitoyl phosphatidylcholine, DPPC, alone, and (b) DPPC $+50 \mathrm{~mol} \%$ cholesterol. The position, $n$, of spin-labelling in the $s n-2$ chain is indicated in the figure. The natural logarithm of the data is plotted.

shown). Consequently $T_{2 \mathrm{M}}$ for 10-PCSL is very much reduced at all temperatures, and the temperature dependence arising from chain dynamics is attenuated. Possibly, weaker spin-spin interactions may also affect, to some extent, the values of $T_{2 \mathrm{M}}$ for labels at other positions of spin labelling in membranes of DPPC alone. Spin-spin broadening is not evident in any of the samples containing $50 \mathrm{~mol} \%$ cholesterol.

\subsection{Echo-detected EPR absorption spectra}

Fig. 3 shows echo-detected field-swept absorption EPR spectra of 14-PCSL in bilayer membranes of dipalmitoyl phosphatidylcholine $+50 \mathrm{~mol} \%$ of cholesterol recorded at $190 \mathrm{~K}$ for different fixed values of the echo delay time, $\tau$. The spectral amplitudes decay with increasing $\tau$ according to the phase-memory time, $T_{2 \mathrm{M}}$ (cf. Fig. 2). However, it is clear from the changing lineshapes that $T_{2 \mathrm{M}}$ varies across the spectrum. This anisotropic phase relaxation, which is characteristic of smallamplitude rotational motions [8,9], is best seen when

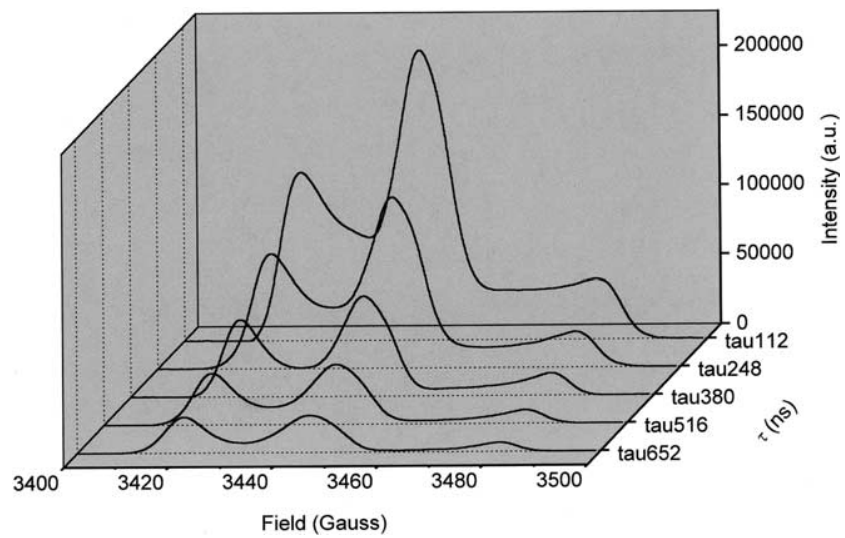

Fig. 3. Echo-detected EPR absorption lineshapes of 14-PCSL spin label in bilayer membranes of dipalmitoyl phosphatidylcholine +50 $\mathrm{mol} \%$ cholesterol at $190 \mathrm{~K}$ for various values of the echo delay time, $\tau$.

spectra are normalised to the amplitude at a stationary position corresponding to an angular turning point.

Fig. 4 gives the echo-detected spectra from the different $n$-PCSL in membranes of DPPC, with and without $50 \mathrm{~mol} \%$ cholesterol, recorded at $190 \mathrm{~K}$. For each sample and label position the spectra are normalised to the low-field outer peak, i.e., to the parallel turning point of the $M_{\mathrm{I}}=+1$ manifold. With this representation the preferential phase relaxation in the intermediate spectral regions at low and high field is more precisely defined. As $\tau$ increases, the position of minimum intensity in the low- and high-field regions moves because phase relaxation comes to dominate over other features that determine the shape of the static powder pattern. The position of the minimum for the largest $\tau$ can be chosen to quantitate the spectral lineheight that is most sensitive to rotational motion. Here we chose the positions of the low- and high-field minima in the spectrum of 5-PCSL in DPPC at $150 \mathrm{~K}$, with $\tau=780 \mathrm{~ns}$. For the other spin labels, $n$-PCSL, which have slightly different outer hyperfine splittings, the diagnostic spectral regions at low and high field are chosen similarly (see Fig. 4). These two positions, relative to the central peak, are then used consistently to analyse all samples with a given spin label.

\subsection{Anisotropic phase relaxation-rotational motion}

Fig. 5 gives the diagnostic lineheight ratios $\mathrm{L}^{\prime \prime} / \mathrm{L}$ and $\mathrm{H}^{\prime \prime} / \mathrm{H}$, at low and high field, respectively, as a function of the echo delay time, $\tau$. $\mathrm{L}^{\prime \prime}$ and $\mathrm{H}^{\prime \prime}$ are the lineheights at the intermediate regions, and $\mathrm{L}$ and $\mathrm{H}$ are those at the outer turning points in the spectrum. There are obvious parallels with the lineheight-ratio analysis that is routinely used in saturation transfer EPR [6]. Data are given in Fig. 5 for spin-label positions, $n=5$ and $n=14$, and for membrane samples with and without $50 \mathrm{~mol} \%$ cholesterol. To within the experimental scatter, the dependence on $\tau$ corresponds to an exponential decay. 


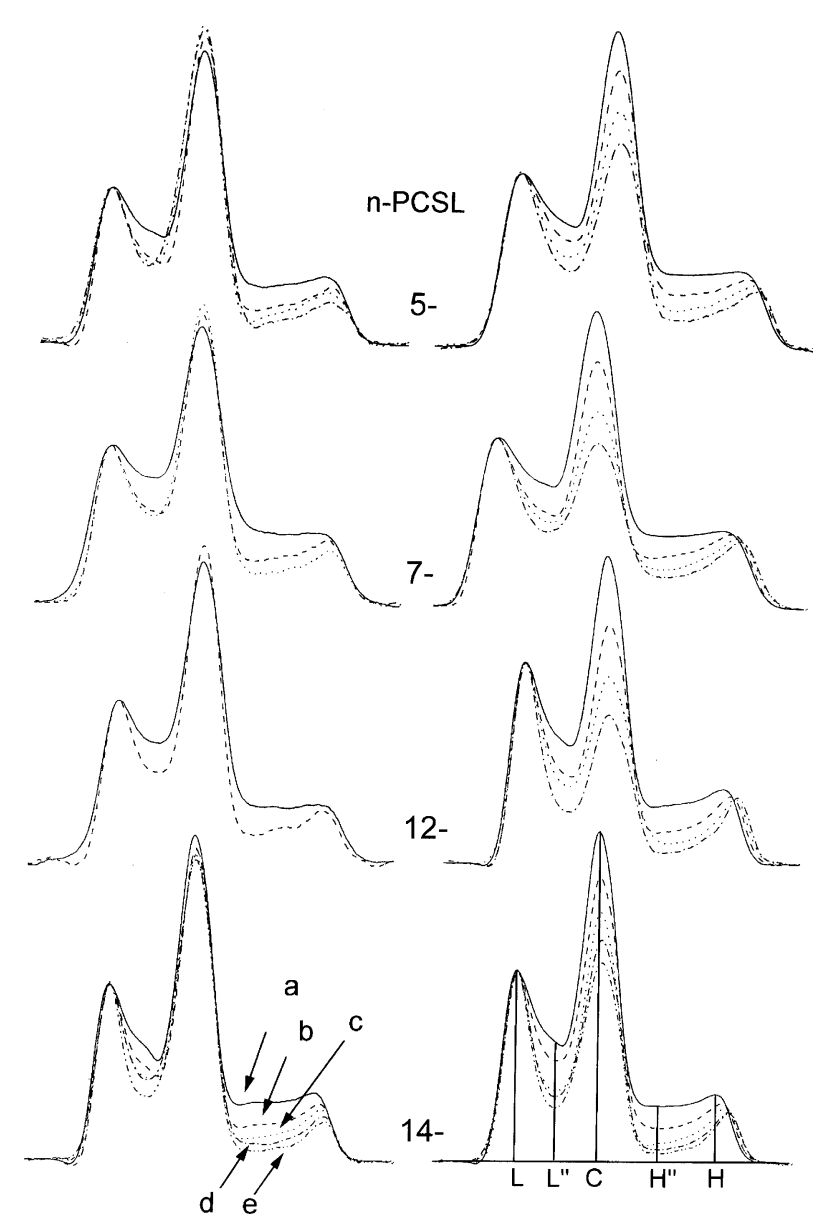

Fig. 4. Echo-detected EPR absorption lineshapes of $n$-PCSL spin labels in bilayer membranes of DPPC (left-hand side) and of DPPC $+50 \mathrm{~mol} \%$ cholesterol (right-hand side) at $190 \mathrm{~K}$ for different positions, $n$, of the spin label in the $s n-2$ chain. For each sample and temperature, the spectra at different values of $\tau$ are normalised to the low-field peak height, L. The values of the echo delay time, $\tau$, are: (a) $108 \mathrm{~ns}$; (b) $244 \mathrm{~ns}$; (c) $380 \mathrm{~ns}$; (d) $516 \mathrm{~ns}$; (e) $652 \mathrm{~ns}$.

A clear difference in decay rate of the lineheight ratios between samples with and without cholesterol is seen in Fig. 5. Also, the results indicate a dependence on spin-label position, at least for membranes containing cholesterol.

The lineheight ratios $\mathrm{L}^{\prime \prime} / \mathrm{L}$ and $\mathrm{H}^{\prime \prime} / \mathrm{H}$, because of their spectral positions, are diagnostic of rotational motion of the nitroxide $z$-axis, i.e., rotations around the $x$ - and/or $y$-axes. Sensitivity to rotation about the $z$-axis is found only in the central region of the spectrum at 9GHz EPR frequencies (cf. $[14,15])$. The most dramatic effects of cholesterol on the echo-detected EPR spectra are actually found in the central region of the spectrum (see Fig. 4). The intensity of the central peak decreases much more rapidly with increasing $\tau$ for samples containing $50 \mathrm{~mol} \%$ cholesterol than for those without cholesterol, at all spin-label positions. Fig. 5 includes data on the ratio, $\mathrm{C} / \mathrm{L}$, of the amplitude of the central peak, C, in the echo-detected spectra to that of the low-

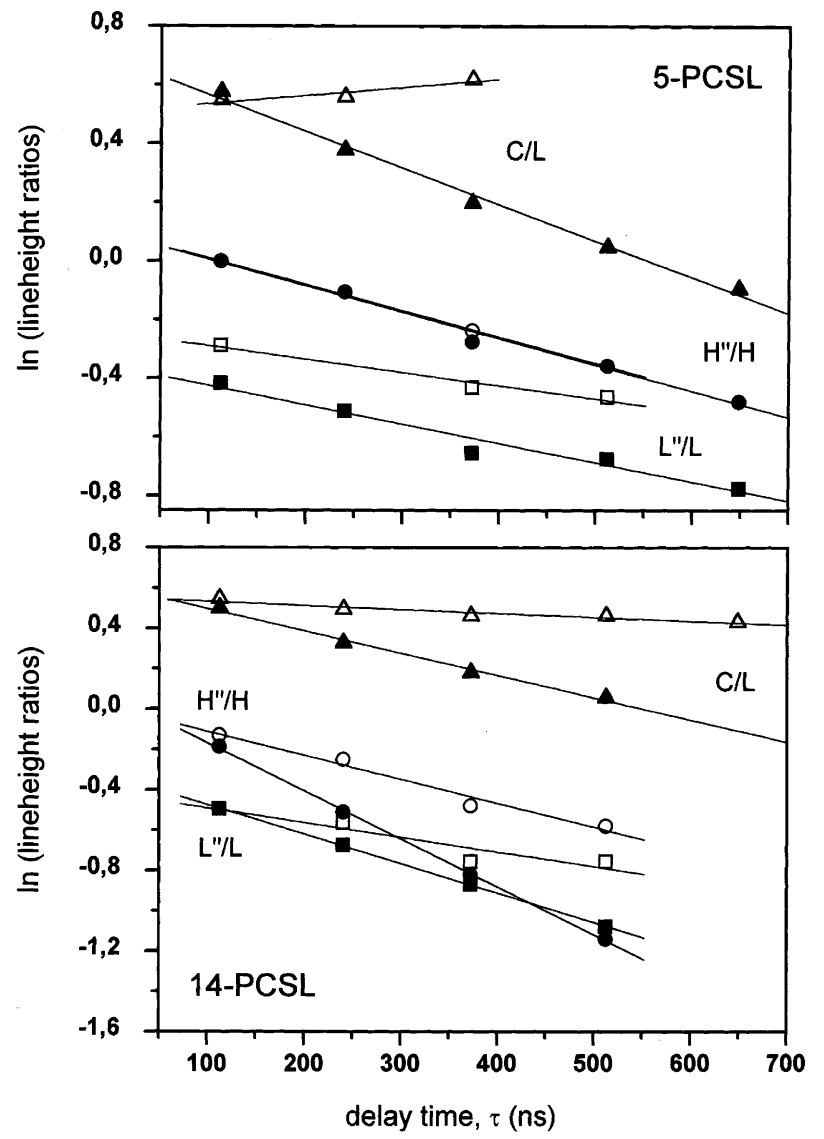

Fig. 5. Dependence of the lineheight ratios $\mathrm{L}^{\prime \prime} / \mathrm{L}(\square)$ and $\mathrm{H}^{\prime \prime} / \mathrm{H}(\mathrm{O})$, in the low- and high-field regions, respectively, and the central lineheight $\mathrm{C} / \mathrm{L}(\triangle)$ normalised to that at low field, on echo delay time, $\tau$. Upper panel: 5-PCSL, and lower panel: 14-PCSL, spin labels in membranes of DPPC, with (solid symbols) and without (open symbols) $50 \mathrm{~mol} \%$ cholesterol, at $150 \mathrm{~K}$. The lineheights, $\mathrm{L}^{\prime \prime}$ and $\mathrm{H}^{\prime \prime}$, are measured at intermediate positions, corresponding to maximum angular spectral dispersion, and $\mathrm{L}$ and $\mathrm{H}$ at the outer maxima, corresponding to angular turning points (see text). $\mathrm{C}$ is the height of the central peak. All lineheight ratios are plotted as the natural logarithm. Solid lines are linear regressions.

field outer peak, L. Again data are given for 5-PCSL and 14-PCSL in membranes with and without cholesterol at $150 \mathrm{~K}$. In both cases, the $\mathrm{C} / \mathrm{L}$ ratio decreases markedly with increasing $\tau$ for samples of DPPC +50 $\mathrm{mol} \%$ cholesterol, whereas it remains comparatively constant for samples of DPPC alone. As for the $\mathrm{H}^{\prime \prime} / \mathrm{H}$ and $\mathrm{L}^{\prime \prime} / \mathrm{L}$ lineheight ratios, the $\mathrm{C} / \mathrm{L}$ ratios shown in Fig. 5 depend exponentially on the echo delay time, $\tau$.

Fig. 6 gives the gradients, obtained from linear regression, of the dependence of the logarithm of all three lineheight ratios on $\tau$, for membranes with and without $50 \mathrm{~mol} \%$ cholesterol. Data are given as a function of spin-label position, $n$, in the $s n-2$ chain of the $n$-PCSL spin-labelled phosphatidylcholines. The results given for membranes without cholesterol are sparser, because (as already noted) spin-spin broadening for labels closer to the middle of the chain results in very rapid echo decays. From these latter decays it is difficult to obtain an 


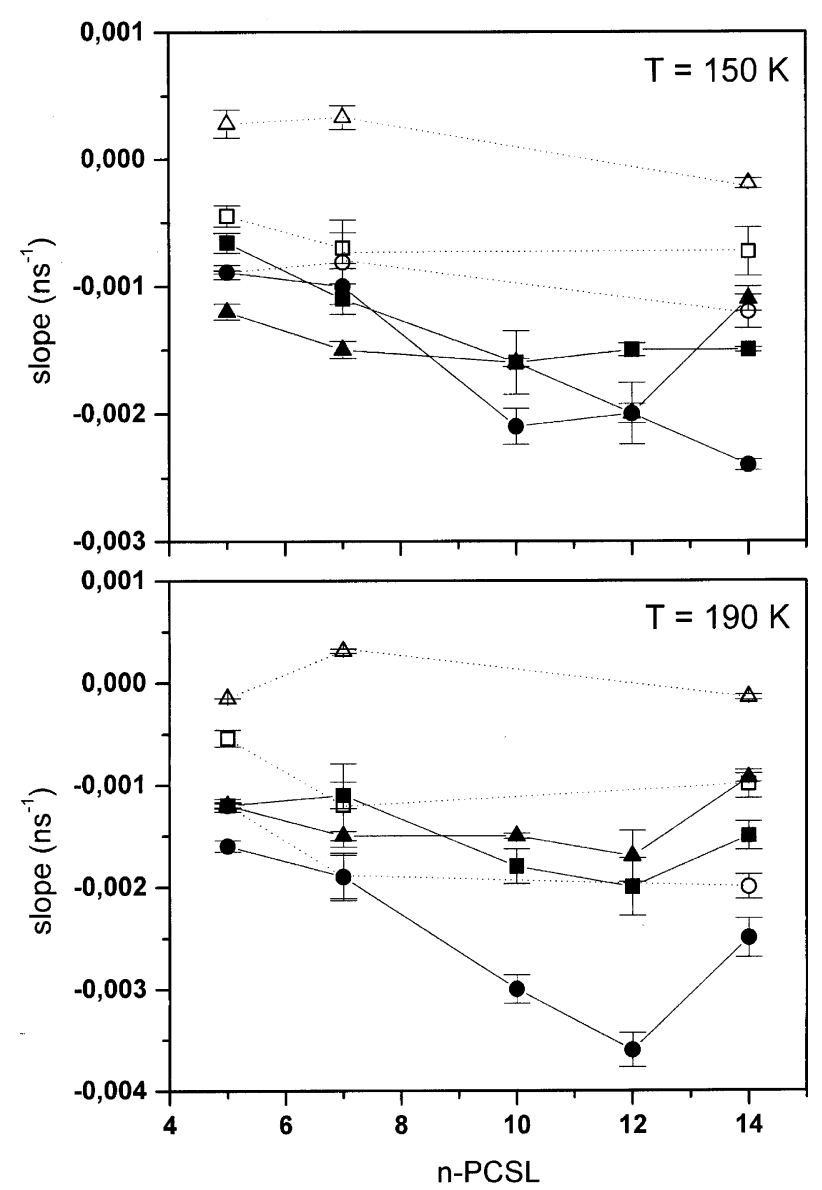

Fig. 6. Slopes from the dependence of $\ln ($ lineheight ratio) on echo delay time, $\tau$ (see Fig. 5), for $n$-PCSL spin labels in membranes of DPPC, with (solid symbols) and without (open symbols) $50 \mathrm{~mol} \%$ cholesterol. Data for lineheight ratios $\mathrm{L}^{\prime \prime} / \mathrm{L}(\square), \mathrm{H}^{\prime \prime} / \mathrm{H}(\mathrm{O})$, and $\mathrm{C} / \mathrm{L}$ $(\triangle)$ are given as a function of spin-label position, $n$, in the $s n-2$ chain. Upper panel: $T=150 \mathrm{~K}$; lower panel: $T=190 \mathrm{~K}$.

accurate $\tau$-dependence because the number of $\tau$-values is severely limited. Nevertheless, systematic differences can be seen in the positional $n$-dependence, between samples with and without cholesterol.

\section{Discussion}

\subsection{Phase memory time, $T_{2 M}$}

The temperature dependence of $T_{2 \mathrm{M}}$ given in Fig. 2 reveals that all measurements are made in the slow motional regime, in which the phase-memory relaxation time increases with increasing rotational correlation time, $\tau_{\mathrm{R}}$, if the rotation is not restricted in amplitude. The minimum in $T_{2 \mathrm{M}}$ before the increase in the fast motional regime is not reached at the highest temperature for which measurements could be made.

Schwartz et al. [7] have made theoretical calculations of the limiting phase-memory times, $T_{\mathrm{M}}^{\infty}$, that are obtained from echoes at long delay times, for rotation of unrestricted amplitude. In the slow motional regime they find that: $T_{\mathrm{M}}^{\infty} \propto \tau_{\mathrm{R}}^{b}$ with exponents $b \cong 1 / 2,2 / 3$ and 1 for Brownian, free and jump rotation, respectively, with isotropic motion. Dzuba and colleagues [9] have proposed a model involving relatively rapid librational motions of limited amplitude to interpret the behaviour in glassy solvents. The phase-memory time then depends both on rotational correlation time, $\tau_{\mathrm{R}}$, and on the restricted angular amplitude of libration, $\overline{\theta^{2}}$. However, when the librational amplitude is determined from the decrease in outer ${ }^{14} \mathrm{~N}$-hyperfine splitting, $A_{z z}$, the resulting correlation times of small spin labels display an anomalous temperature dependence, decreasing with decreasing temperature, in glassy matrices ([11], and see also [16]).

The rotational motion of the spin-labelled lipids that we have used is undoubtedly not only anisotropic but also of limited amplitude, especially in the low-temperature phases of bilayer membranes that are studied here. It is therefore clear that the temperature dependence of $T_{2 \mathrm{M}}$ in Fig. 2 is determined both by the rotational/torsional amplitude and by the rotational rate, i.e., correlation time. The angular amplitude of this motion decreases with decreasing temperature, and most probably the motional rate is at least partially in the slow-motion regime. Indeed, Kirilina et al. [11] find it necessary to include a slow wobbling motion, in addition to rapid libration, in order to explain their echodetected spectra at long delay times. Both decreasing amplitude and increasing correlation time in the slowmotion regime cause $T_{2 \mathrm{M}}$ to increase with decreasing temperature, as is observed in Fig. 2.

\subsection{Activation energies}

If it is assumed that the rotational rate obeys an Arrhenius law in the slow-motion regime, i.e., $\tau_{\mathrm{R}}=\tau_{\mathrm{R}, 0} \exp \left(E_{\mathrm{a}} / R T\right)$, then the gradient of $\ln \left(T_{2 \mathrm{M}}\right)$ against $\ln (1 / T)$ gives a measure of the activation energy, $E_{\mathrm{a}}$. Values obtained from the linear regions in the Arrhenius plots, which are found at the higher temperatures of the range given in Fig. 2, are presented in Table 1 . With the functional dependence on rotational correlation time that was given above for unrestricted rotation in the slow motional regime [7], these values are given by $\left(E_{\mathrm{a}} / R\right) \times b$. For jump rotation, this is equal to the activation energy, $E_{\mathrm{a}} / R$, and for Brownian rotation, the activation energy is twice this value. With the exception of 7-PCSL, rather similar values are obtained for the effective activation energies at different label positions, in the absence of cholesterol (see Table 1 and cf. upper panel of Fig. 2). In contrast, for membranes containing $50 \mathrm{~mol} \%$ cholesterol, a decrease in effective activation energy with spin-label chain position is found towards the terminal methyl end of the chain, i.e., for 12-PCSL and 14-PCSL in Table 1. (Again 7-PCSL is an 
Table 1

Activation energies, $E_{\mathrm{a}}$, from the gradients of $\ln \left(T_{2 \mathrm{M}}\right)$ vs. $\ln (1 / T)$ for $n$-PCSL spin labels in DPPC membranes with and without $50 \mathrm{~mol}^{\%}$ cholesterol ${ }^{\mathrm{a}}$

\begin{tabular}{lll}
\hline Spin label & $\left(E_{\mathrm{a}} / R\right) \times b$ & \\
\hline & - chol & + chol \\
5-PCSL & $4.8 \pm 0.3$ & $5.3 \pm 0.9$ \\
7-PCSL & $2.2 \pm 0.2$ & $3.0 \pm 0.2$ \\
10-PCSL & $(4.2)$ & $3.9 \pm 0.1$ \\
12-PCSL & $4.6 \pm 0.8$ & $3.1 \pm 0.3$ \\
14-PCSL & $4.4 \pm 0.1$ & $2.5 \pm 0.3$ \\
\hline
\end{tabular}

${ }^{\mathrm{a}}$ Taken from the linear region in the slow-motion regime $(T>200 \mathrm{~K})$ of Fig. 2.

exception.) At the position of the rigid cholesterol ring system, the chain libration is restricted, whereas, further down the chain, the cholesterol molecule is less bulky and more flexible, which results in a decreased chainpacking density and sterically less restricted motion. On the other hand, in the absence of cholesterol, the chains are rather uniformly close-packed in the low-temperature phase. The difference is greatest at the $\mathrm{C}-14$ chain position, which corresponds to the extreme end of the cholesterol molecule.

For comparison with the data given in Table 1, a value of $\left(E_{\mathrm{a}} / R\right) \times b \approx 7.5$ has been obtained for a small spin label, TEMPONE, in a glassy solvent [17], and $\left(E_{\mathrm{a}} / R\right) \times b=2.5 \pm 0.5$ for a spin-labelled peptide in a glassy matrix [16]. Jump diffusion (i.e., $b=1$ ) was favoured over Brownian diffusion in the former case [7], whereas Brownian diffusion (i.e., $b=1 / 2$ ) gave a better fit in the latter case [16]. Small-amplitude oscillations, rather than jump diffusion, are expected for the librational motions in the low-temperature phase of the lipid membranes that are studied here (see also later). Compared with the small-amplitude, unrestricted Brownian diffusion of the spin-labelled peptide in a glassy medium, the effective activation energies in Table 1 are mostly larger (but smaller than the jump diffusion of TEMPONE). This correlates with the restricted nature of the librational motion of the lipid chains in membranes at low temperature. Only for 14-PCSL in cholesterol-containing membranes, for which the librational motion is expected to be less restricted (as already noted), is the effective activation energy comparable to the Brownian case.

At lower temperatures, $T_{2 \mathrm{M}}$ for 14-PCSL (and also for 10 -PCSL and 12-PCSL in DPPC $+50 \mathrm{~mol} \%$ cholesterol) passes through a maximum and then decreases as the temperature is lowered further. This biphasic response, which is found also for small spin labels in glassy solvents [17], was originally ascribed to the onset of a solid-state mechanism for relaxation. Subsequently Dzuba et al. [18] attributed the solid-state process to thermally activated rotation of methyl groups in the nitroxide moiety. In this case, the methyl group rotation is in the fast motional regime, even at low temperature in the semi-solid state. Similar effects have also been observed for an end-labelled tetraproline hexapeptide in a glass-forming matrix and interpreted in the same way ([16] and references therein). This interpretation should apply also here, although the relaxation will be somewhat less efficient because of the reduced number of methyl groups for a doxyl relative to the usual piperidine and pyrrolidine nitroxides. The activation energy for rotation of the methyl groups is much smaller than for the restricted librational motion of the lipid chains (compare the low- and high-temperature regions in Fig. 2). This is because of the approximately spherical shape of the methyl group, which engenders no steric hindrance to rotation.

\subsection{Echo-detected lineshapes}

The anisotropic phase-memory relaxation that is evident from the dependence of the echo-detected lineshapes on $\tau$ arises from small-amplitude oscillatory motions of the spin-labelled chain segments. Maximum effects are seen in those regions of the spectrum for which the change in spectral field position with angular orientation is greatest (see Figs. 3 and 4). These are in the intermediate positions at low and high field, where the angular variation depends primarily on the orientation, $\theta$, of the nitroxide $z$-axis to the static magnetic field. There are obvious analogies with $\mathrm{CW}$ saturation transfer EPR, where sensitivity to slow rotational motion (on the $T_{1}$-timescale) is quantitated in terms of the ratio of lineheights at the intermediate field positions to those at the stationary turning points [6].

Although strictly applicable only to the fast-motion regime, the analysis of Kirilina et al. [11] can be used to give a qualitative interpretation of the change in lineshape with increasing $\tau$. The dependence of the echo intensity on field orientation, $\theta$, is given for small-amplitude librations by

$V(2 \tau)=\exp \left(-2 \tau / T_{2}^{0}\right) \exp \left\lfloor-2 \tau R^{2}(\theta) J(0)\right\rfloor$,

where, for the hyperfine manifold with nuclear magnetic quantum number $M_{\mathrm{I}}$,

$$
\begin{aligned}
R^{2}(\theta)= & \frac{1}{4}\left[\beta_{e} H\left(g_{y y}-g_{z z}\right)\right. \\
& \left.+\frac{M_{I}\left(A_{y y}^{2}-A_{z z}^{2}\right)}{\sqrt{A_{y y}^{2} \sin ^{2} \theta+A_{z z}^{2} \cos ^{2} \theta}}\right]^{2} \sin ^{2} 2 \theta
\end{aligned}
$$

with $g$-tensor $\mathbf{g}=\left(g_{x x}, g_{y y}, g_{z z}\right)$ and ${ }^{14} \mathrm{~N}$-hyperfine tensor $\mathbf{A}=\left(A_{x x}, A_{y y}, A_{z z}\right) ; \beta_{e}$ is the Bohr magnetron and $H$ is the static magnetic field strength. For simplicity, rotation is assumed to take place around the nitroxide $x$-axis. Orientation-independent contributions to dephasing of 
the transverse magnetisation are given by the intrinsic $T_{2}$-relaxation time, $T_{2}^{0}$. In Eq. (1), it is assumed that contributions from the spectral density at zero frequency, $J(0)$, dominate over those at the electron Larmor frequency, as is usually the case for transverse relaxation.

Fig. 7 gives the angular dependence of $R^{2}(\theta)$ for a nitroxide spin label. Significant contributions are found for the $M_{\mathrm{I}}= \pm 1{ }^{14} \mathrm{~N}$-manifolds. The $R^{2}(\theta)$ function reaches a maximum at $65^{\circ}$ and $69^{\circ}$ for the low-field and high-field regions, respectively, i.e., considerably closer to $\theta=90^{\circ}$ than to $\theta=0^{\circ}$. To make a simple model prediction of the effects on EPR lineshape we neglect $g$-value anisotropy and assume that the hyperfine anisotropy has the approximate form: $A(\theta)=\left(A_{z z}-\right.$ $\left.A_{y v}\right) \cos ^{2} \theta+A_{y y}$. The powder lineshape is then given by $L_{0}(\sigma)=\frac{1}{2} \sigma^{-1 / 2}$ with a normalised scan parameter $\sigma=\left\lfloor A(\theta)-A_{y y}\right\rfloor /\left(A_{z z}-A_{y y}\right)$ (see e.g., [19]). The model

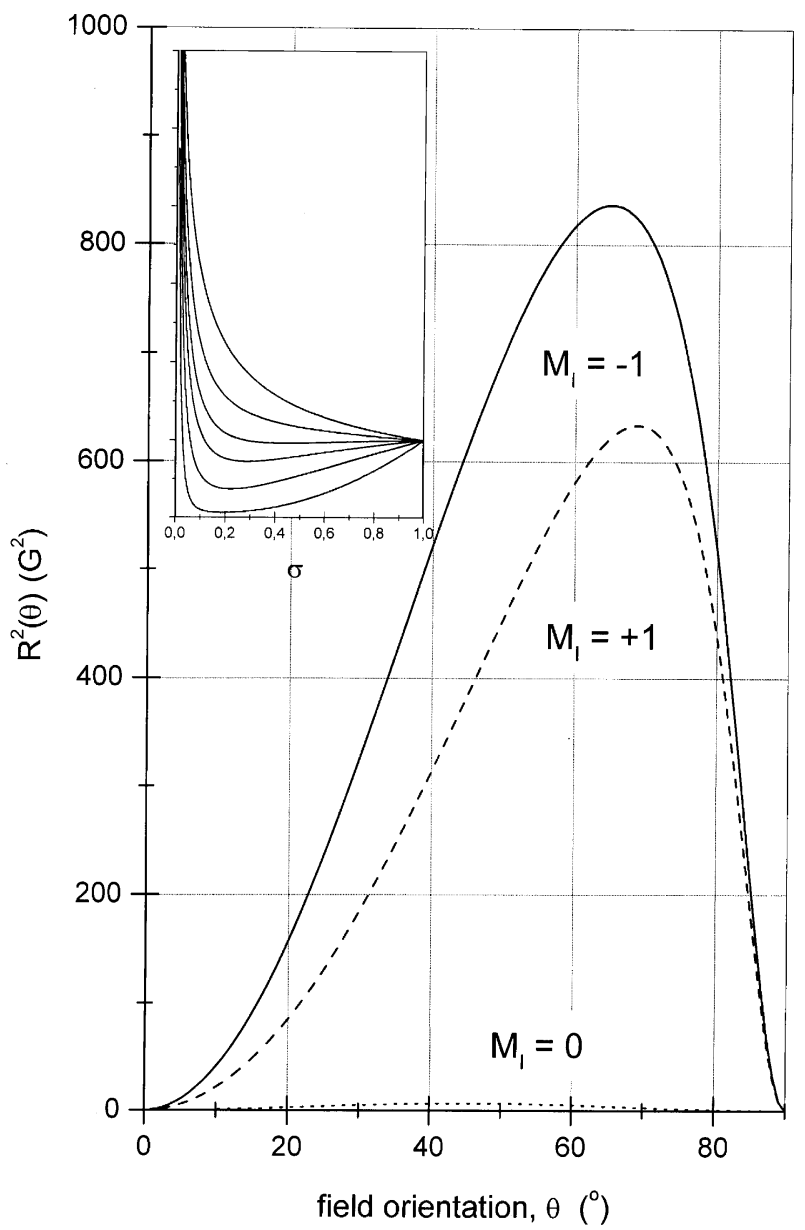

Fig. 7. Dependence of the inverse phase-memory time, $1 / T_{2 \mathrm{M}}(\theta)$ $\left(\equiv R^{2}(\theta) \times J(0)\right)$ on the orientation, $\theta$, of the magnetic field relative to the nitroxide $z$-axis. Calculations are made from Eqs. (1) and (2), with $g_{y y}=2.006, g_{z z}=2.003, A_{y y}=6 \mathrm{G}$, and $A_{z z}=33 \mathrm{G}$. Inset: simulated model echo-detected axial absorption lineshapes with increasing values of $\tau$ (top to bottom) according to Eqs. (3) and (4). The uppermost lineshape corresponds to $\tau=0$. echo-detected lineshape in the presence of angular oscillation is then given from Eq. (1) by

$L(\sigma, \tau)=\frac{1}{2} \sigma^{-1 / 2} \exp \left[-2 \tau R^{2}(\sigma) J(0)\right]$,

where, from Eq. (2),

$R^{2}(\sigma)=\frac{1}{4} \frac{M_{\mathrm{I}}^{2}\left(A_{y y}+A_{z z}\right)^{2}}{\left[\sigma+A_{y y} /\left(A_{z z}-A_{y y}\right)\right]^{2}}\left[1-(2 \sigma-1)^{2}\right]$.

The inset in Fig. 7 gives predictions from Eqs. 3 and 4 for the dependence of the model lineshape on interpulse spacing, $\tau$. Greatest dependence on $\tau$ is found at intermediate spectral positions $\sigma$ that are considerably closer to the $\sigma_{\perp}=0$ discontinuity than to the $\sigma_{/ /}=1$ shoulder of the powder pattern. It is this feature that was used to define the lineheight, $\mathrm{L}^{\prime \prime}$ or $\mathrm{H}^{\prime \prime}$, at intermediate field for the diagnostic ratios given in Fig. 5. Note that, from Eq. (3), these diagnostic lineheight ratios, at fixed field positions $\sigma$, should depend exponentially on the echo delay time, $\tau$. In the librational model, for a fixed value of $\tau$, they should also depend exponentially on the spectral density $J(0)$, and hence on the rotational amplitude $\left(\overline{\theta^{2}}\right)$ and correlation time $\left(\tau_{D}\right)$ according to $\overline{\theta^{2}} \tau_{D}$ for the rapid librational model [11].

The inset in Fig. 7 displays a remarkable qualitative resemblance to the high-field region of the echo-detected spectra given in Figs. 3 and 4. This explains the physical origin of the angular dependence of the echo decays, but, of course, does not establish the validity of the fastmotion analysis. Kirilina et al. [11] have obtained good agreement between experiment and spectral simulation with the librational model for small spin labels in glasses. As noted above, however, an additional slow wobbling motion was required, and the temperature dependence obtained for the librational spectral density was counter-intuitive. Saxena and Freed [16] have obtained only a semi-quantitative agreement with experiment for the echo-detected lineshapes of a spin-labelled peptide in a glycerol-water-trifluoroethanol glass by assuming anisotropic Brownian diffusion of unlimited amplitude. The anisotropic rotational diffusion coefficients involved were on the border of the slow motional regime and 100 times slower. As already mentioned, the spin-labelled lipid motion studied here is anisotropic and of limited amplitude, and it must also contain components in the slow regime, as established in the two aforementioned simulation studies.

\subsection{Diagnostic lineheight ratios $L^{\prime \prime} / L$ and $H^{\prime \prime} / H$}

The lineheight ratios for the low-field and high-field manifolds are measured at intermediate positions, where $R^{2}(\theta)$ has its maximum value, relative to the corresponding turning points for which $R^{2}(\theta)=0$. From Eq. (1), it is seen that taking the ratio eliminates the angle-independent contributions to $T_{2 \mathrm{M}}$ that are 
determined by $T_{2}^{0}$. According to Eq. (1), the diagnostic lineheight ratios should depend exponentially on the echo delay time, $\tau$. Fig. 5 shows that this is the case, to within the experimental uncertainties. Therefore, the gradients of $\ln \left(\mathrm{L}^{\prime \prime} / \mathrm{L}\right)$ or $\ln \left(\mathrm{H}^{\prime \prime} / \mathrm{H}\right)$ vs. $\tau$ that are given in Fig. 6 can be used to discuss the dynamic behaviour of the system. They are a direct measure of the spectral density $J(0)$ (see Eq. (1)), which depends on the rotational amplitude and correlation time.

For membranes without cholesterol, the slopes obtained from the $\tau$-dependence of all three lineheight ratios do not vary greatly with spin-label position in the acyl chain, particularly at $150 \mathrm{~K}$. This might be expected for librational chain motions in the low-temperature phase of membranes composed from saturated lipids. The lipid chains are closely packed throughout their length, and cumulative torsional rotations are absent. In membranes containing cholesterol, the situation is rather different (see Fig. 6). The absolute values of the $\tau$-gradients are larger than those in the absence of cholesterol, and a pronounced dependence is found on the chain position at which the spin label is attached. Cholesterol clearly disrupts chain close-packing in the low-temperature phase of the lipid membranes at $150 \mathrm{~K}$, increasing the amplitude of rotational librations and allowing them to become cumulative down the chain. On increasing the temperature to $190 \mathrm{~K}$, a biphasic dependence of the $\mathrm{L}^{\prime \prime} / \mathrm{L}$ and $\mathrm{H}^{\prime \prime} / \mathrm{H}$ gradients on chain position is found towards the terminal methyl end of the chain (see lower panel in Fig. 6). This trend (already seen in $\mathrm{C} / \mathrm{L}$ at $150 \mathrm{~K}$ ) could be explained by a change in correlation time coming to dominate over changes in amplitude at the $n=14$ position of the chain. Note that the $\mathrm{C}-14$ position of the lipid chain is situated below the rigid steroid nucleus of cholesterol in the membrane, and hence is likely to have an increased rotational freedom in the presence of the intercalated steroid.

\subsection{Central lineheights, $C / L$}

The behaviour of the central peak in the echodetected spectra is of considerable importance because of the pronounced influence of cholesterol on this lineheight as a function of $\tau$ (see Fig. 4). Previously it has been found that diffusion-controlled spin-spin interactions can lead to such effects for small spin labels [20]. The preferential effect on the central manifold arises because of its narrow spectral dispersion. Far more spin packets are excited by a microwave pulse of given width than in the outer manifolds, e.g., at the L-position, where angular dispersion and consequently spectral dispersion is much larger.

Spin-spin interactions are unlikely to be the explanation for the influence of cholesterol because, where appreciable, spin-spin interactions are much larger in the absence of cholesterol than in its presence. For 10-
PCSL in membranes of DPPC alone, $T_{2 \mathrm{M}}$ measured on the central peak is atypically low because of static spinspin interactions, but this does not give rise to a preferential decay in the central region of the echo-detected spectra (data not shown). Further, diffusion-controlled spin-spin interactions are unlikely to play an important role because translational diffusion of the spin-labelled lipids is expected to be almost stopped in the low-temperature phases of these membranes. Also the temperature dependence of $\mathrm{C} / \mathrm{L}$ is not that expected for a diffusion-controlled process. Where there are differences between 150 and $190 \mathrm{~K}$ in the slopes for $\mathrm{C} / \mathrm{L}$, the absolute values are, if anything, smaller at the higher temperature (see Fig. 6).

The most likely explanation for the preferential phase relaxation in the central region of spectra from membranes containing cholesterol is that this spectral region (typified by the $\mathrm{C} / \mathrm{L}$ ratio) is sensitive to axial rotational motions, in addition to the off-axis motions to which the outer spectral regions (i.e., $\mathrm{L}^{\prime \prime} / \mathrm{L}$ and $\left.\mathrm{H}^{\prime \prime} / \mathrm{H}\right)$ are exclusively sensitive $[14,15]$. As for the $\mathrm{H}^{\prime \prime} / \mathrm{H}$ and $\mathrm{L}^{\prime \prime} / \mathrm{L}$ lineheight ratios, one would then expect an exponential dependence of $\mathrm{C} / \mathrm{L}$ on echo delay time, $\tau$, to arise from this rotational motion, as indeed is observed for the membranes containing cholesterol (see Fig. 5). Therefore, the preferential effect of cholesterol in the low-temperature membrane phases most probably corresponds to an increased torsional oscillation of the lipid chains about the long axis that is not present in membranes without cholesterol. The origin of this increased axial motional freedom is ascribed to irregular chain packing in the presence of the bulky sterol molecule, as evidenced by the absence of any tendency for the spin-label to segregate from the host lipid in the low-temperature phases containing cholesterol. The situation is very different from that in the liquid-ordered phase of cholesterol-containing membranes in which large-amplitude axial rotation of the lipid chains is suppressed for steric reasons [21-23].

\section{Conclusions}

The results of this study indicate the importance of anisotropic rotational oscillations of the lipid chain segments in low-temperature membrane phases. The membrane environment with saturated lipids has certain similarities to that for small spin labels in glassy matrices. Cholesterol inhibits to some extent the off-axis angular oscillations, but strongly enhances the axial torsional motions. It can be speculated that these dynamic properties of the lipids may be important in cryoprotection. Such small-amplitude angular oscillations may enhance the stability of membrane-embedded integral proteins; admixtures with cholesterol could also reduce the tendency to aggregation. In this connection it is interesting 
to note that in our reconstitutions of cytochrome oxidase with saturated phosphatidylcholine, we found that storing over prolonged periods in the lipid gel phase maintained activity when assayed in the higher temperature fluid phase $[24,25]$. On the other hand, activity was lost progressively on incubating in the fluid lipid phase.

\section{Acknowledgments}

R.B., D.M., and L.S. are members of the European COST D22 Action. This work was performed in the framework of the project CIPE-MIA 26-WP3. We thank Birgit Angerstein for spin-label synthesis.

\section{References}

[1] L.J. Berliner (Ed.), Spin Labeling. Theory and Applications, Academic Press, New York, 1976.

[2] D. Marsh, L.I. Horváth, Spin-label studies of the structure and dynamics of lipids and proteins in membranes, in: A.J. Hoff (Ed.), Advanced EPR. Applications in Biology and Biochemistry, Elsevier, Amsterdam, 1989, pp. 707-752.

[3] D. Marsh, Experimental methods in spin-label spectral analysis, in: L.J. Berliner, J. Reuben (Eds.), Biological Magnetic Resonance, vol. 8, Plenum Publishing, New York, 1989, pp. 255-303.

[4] D. Marsh, L.I. Horváth, Structure, dynamics and composition of the lipid-protein interface. Perspectives from spin-labelling, Biochim. Biophys. Acta 1376 (1988) 267-296.

[5] J.H. Freed, Theory of slowly tumbling ESR spectra for nitroxides, in: L.J. Berliner (Ed.), Spin Labeling, Theory and Applications, Academic Press, New York, 1976, pp. 53-132.

[6] D.D. Thomas, L.R. Dalton, J.S. Hyde, Rotational diffusion studied by passage saturation transfer electron paramagnetic resonance, J. Chem. Phys. 65 (1976) 3006-3024.

[7] L.J. Schwartz, A.E. Stillman, J.H. Freed, Analysis of electron spin echoes by spectral representation of the stochastic Liouville equation, J. Chem. Phys. 77 (1982) 5410-5425.

[8] G.L. Millhauser, J.H. Freed, Two-dimensional electron spin echo spectroscopy and slow motions, J. Chem. Phys. 81 (1984) 37-48.

[9] S.A. Dzuba, Yu.D. Tsvetkov, A.G. Maryasov, Echo-detected EPR spectra of nitroxides in organic glasses: model of orientational molecular motions near equilibrium position, Chem. Phys. Lett. 188 (1992) 217-222.

[10] S.A. Dzuba, Libration motion of guest spin probe molecules in organic glasses: CW EPR and electron spin echo study, Spectrochim. Acta A 56 (2000) 227-234.
[11] E.P. Kirilina, S.A. Dzuba, A.G. Maryasov, Y.D. Tsvetkov, Librational dynamics of nitroxide molecules in a molecular glass studied by echo-detected EPR, Appl. Magn. Reson. 21 (2001) 203-221.

[12] L. Kar, E. Neyigner, J.H. Freed, Electron spin resonance and electron spin echo study of oriented multilayers of $\mathrm{L}$ - $\alpha$-dipalmitoylphosphatidylcholine water systems, Biophys. J. 48 (1985) 569-595.

[13] D. Marsh, A. Watts, Spin-labeling and lipid-protein interactions in membranes, in: P.C. Jost, O.H. Griffith (Eds.), Lipid-Protein Interactions, vol. 2, Wiley-Interscience, New York, 1982, pp. 53126.

[14] D. Marsh, Molecular motion in phospholipid bilayers in the gel phase: long axis rotation, Biochemistry 19 (1980) 16321637.

[15] P. Fajer, P.F. Knowles, D. Marsh, Rotational motion of yeast cytochrome oxidase in phosphatidylcholine complexes studied by saturation-transfer electron spin resonance, Biochemistry 28 (1989) 5634-5643.

[16] A. Saxena, J.H. Freed, Two-dimensional electron spin resonance and slow motions, J. Phys. Chem. A 101 (1997) 7998-8008.

[17] A.E. Stillman, L.J. Schwartz, J.H. Freed, Direct determination of rotational correlation time by electron spin echoes, J. Chem. Phys. 73 (1980) 3502-3503.

[18] S.A. Dzuba, A.G. Maryasov, K.M. Salikov, Yu.D. Tsvetkov, Superslow rotations of nitroxide radicals studied by pulse EPR spectroscopy, J. Magn. Reson. 58 (1984) 95-117.

[19] D. Marsh, L.I. Horváth, Influence of Heisenberg spin exchange on conventional and phase-quadrature EPR lineshapes and intensities under saturation, J. Magn. Reson. 97 (1992) 13-26.

[20] Yu.V. Toropov, S.A. Dzuba, Yu.D. Tsvetkov, V. Monaco, F. Formaggio, M. Crisma, C. Toniolo, J. Raap, Molecular dynamics and spatial distribution of TOAC spin-labeled studied in glassy liquid by echo-detected EPR spectroscopy, Appl. Magn. Reson. 15 (1998) 237-246.

[21] B.J. Gaffney, D. Marsh, High-frequency spin-label EPR of nonaxial lipid ordering and motion in cholesterol-containing membranes, Proc. Natl. Acad. Sci. USA 95 (1998) 1294012943.

[22] V.A. Livshits, D. Marsh, Simulation studies of high-field EPR spectra of spin-labeled lipids in membranes, J. Magn. Reson. 147 (2000) 59-67.

[23] D. Kurad, G. Jeschke, D. Marsh, Spin-label HF-EPR of lipid ordering in cholesterol-containing membranes, Appl. Magn. Reson. 21 (2001) 469-481.

[24] P.F. Knowles, A. Watts, D. Marsh, Spin label studies of lipid immobilization in dimyristoylphosphatidylcholine-substituted cytochrome oxidase, Biochemistry 18 (1979) 4480-4487.

[25] P. Fajer, D. Marsh, Sensitivity of saturation transfer ESR spectra to anisotropic rotation. Application to membrane systems, J. Magn. Reson. 51 (1983) 446-459. 